\title{
Matter-Wave Diffraction from a Periodic Array of Half Planes
}

\author{
Ju Hyeon Lee, ${ }^{1}$ Lee Yeong Kim, ${ }^{2}$ Yun-Tae Kim, ${ }^{3}$ Chang Young Lee, ${ }^{4}$ \\ Wieland Schöllkopf, ${ }^{5, *}$ and Bum Suk Zhao (조범석) ${ }^{1,2, \uparrow}$ \\ ${ }^{1}$ Department of Chemistry, Ulsan National Institute of Science and Technology (UNIST), Ulsan 44919, Korea \\ ${ }^{2}$ Department of Physics, Ulsan National Institute of Science and Technology (UNIST), Ulsan 44919, Korea \\ ${ }^{3}$ Department of Biomedical Engineering, Ulsan National Institute of Science and Technology (UNIST), Ulsan 44919, Korea \\ ${ }^{4}$ Department of Chemical Engineering, Ulsan National Institute of Science and Technology (UNIST), Ulsan 44919, Korea \\ ${ }^{5}$ Fritz-Haber-Institut der Max-Planck-Gesellschaft, Faradayweg 4-6, 14195 Berlin, Germany
}

(Received 23 June 2018; revised manuscript received 2 October 2018; published 31 January 2019)

\begin{abstract}
We report on reflection and diffraction of beams of He and $D_{2}$ from square-wave gratings of a $400-\mu \mathrm{m}$ period and strip widths ranging from 10 to $200 \mu \mathrm{m}$ at grazing-incidence conditions. In each case we observe fully resolved matter-wave diffraction patterns including the specular reflection and diffracted beams up to the second diffraction order. With decreasing strip width, the observed diffraction efficiencies exhibit a transformation from the known regime of quantum reflection from the grating strips to the regime of edge diffraction from a half-plane array. The latter is described by a single-parameter model developed previously to describe phenomena as diverse as quantum billiards, scattering of radio waves in urban areas, and reflection of matter waves from microstructures. Our data provide experimental confirmation of the widespread model. Moreover, our results demonstrate that neither classical reflection nor quantum reflection are essential for reflective diffraction of matter waves from a structured solid, but it can result exclusively from half-plane edge diffraction.
\end{abstract}

DOI: 10.1103/PhysRevLett.122.040401

Diffraction from a periodic array of half planes as depicted schematically in Fig. 1(a) is a wave-optical phenomenon that plays an important role in various fields of science and technology, including optics [1], statistical quantum mechanics [2-5], atom optics [6,7], and ultrahigh frequency (UHF) communication in urban areas [8-10]. For instance, in models of UHF radio-wave propagation, building blocks are approximated by arrays of absorbing half planes at near grazing incidence $[9,10]$. Moreover, pseudointegrable solutions found in polygonal quantum billiards have been modeled by scattering of waves from infinite periodic half-plane arrays at grazing incidence with respect to the plane formed by the half-plane edges [3,4]. Furthermore, in atom optics, reflection of ultracold atoms from a periodically ridged surface was observed [6] and attributed to diffraction of the atomic de Broglie waves from an array of half planes. It was therefore dubbed the "Fresnel diffraction mirror" [6,7].

Consider a plane-wave incident onto an individual half plane [Fig. 1(a)]. The part not blocked by the half plane propagates along its incident direction towards the next half plane. However, the clipping causes edge diffraction: a small part of the (matter) wave propagates in other directions. According to the uncertainty principle, clipping reduces the uncertainty in coordinate space and hence increases the uncertainty in momentum space observed in a diffraction pattern.

The exact solution for the scattering of an electromagnetic plane wave off a periodic array of parallel half planes was first derived by Carlson and Heins in the absence of diffracted beams [11,12]. Subsequently, the theoretical analysis was extended to conditions where a diffracted beam is present $[13,14]$, and later the Walfisch-Bertoni model (also referred to as the diffracting-screens model) was introduced to describe propagation of UHF electromagnetic waves in urban areas $[9,10]$.

Although the wavelengths in those scattering problems vary by 10 orders of magnitude from $0.1 \mathrm{~nm}$ for atomic de Broglie waves to $1 \mathrm{~m}$ for radio waves, a single scalable parameter $u$ is used to model the effect [3],

$$
u=\sqrt{\frac{2 d}{\lambda}} \sin \frac{\theta_{\text {in }}}{2} .
$$
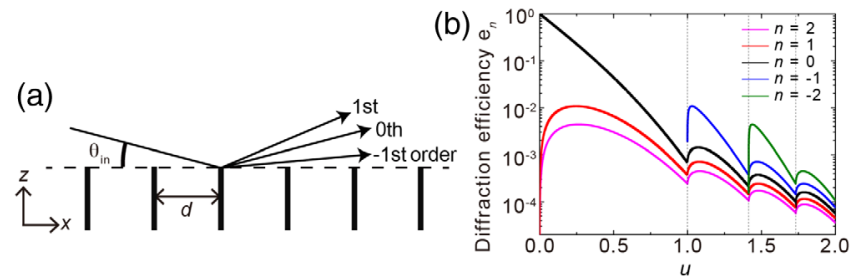

FIG. 1. (a) Schematic of wave scattering from a periodic array of parallel (zero-width) half planes at grazing incidence angle $\theta_{\text {in }}$. The grating plane (dashed horizontal line) is defined by the halfplane edges. (b) Diffraction efficiencies $e_{n}(u)$ for the specular $(n=0)$ and for diffracted beams up to the second order calculated by the Bogomolny-Schmit solution given by Eqs. (2) and (3) [3]. 


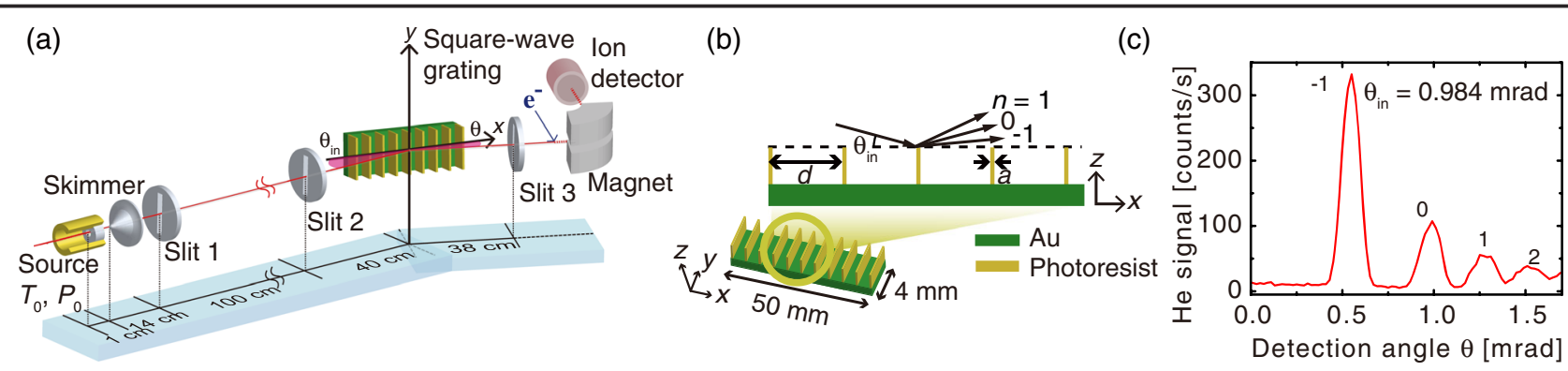

FIG. 2. (a) Schematic of the experimental setup. The grating normal is chosen as the $z$ axis of our coordinate system. Incidence $\theta_{\text {in }}$ and detection angle $\theta$ are measured with respect to the grating surface in the $x z$ plane of incidence. (b) The reflection gratings are 50-mmlong microstructured arrays of 4-mm-long parallel strips made out of 1- $\mu$ m-thick photoresist patterned on a commercial gold mirror. The center-to-center distance of the strips defines the period $d=400 \mu \mathrm{m}$ identical for all gratings used. Four gratings with strip width $a=10,30,100$, or $200 \mu \mathrm{m}$ have been used. For all four gratings, the gold surface between the strips is completely shadowed by the strips for all incidence angles used in this Letter. A sketch of the $a=10 \mu \mathrm{m}$ grating is shown in (b). (c) Representative diffraction pattern for $a=10 \mu \mathrm{m}$ and $\theta_{\text {in }}=0.984 \mathrm{mrad}$ measured by rotating the detector around the $y$ axis and integrating the signal for $8 \mathrm{~s}$ at each angular position.

(Various prefactors such as $\sqrt{2} u[9], 2 u[6]$, and $2 \sqrt{\pi} u[4,7]$ have been used.) Here, the period of the half-plane array is denoted by $d$, while $\lambda$ and $\theta_{\text {in }}$ stand for the wavelength of the incident beam and the incidence angle (measured with respect to the structure surface plane as indicated in Fig. 1), respectively.

Bogomolny and Schmit $[3,4]$ extended the earlier theoretical description to the semiclassical limit of small wavelength and grazing-incidence conditions where, in addition to the specular reflection, multiple diffraction beams propagate above the grating plane [see Fig. 1(a)]. For these conditions, which are well fulfilled in our experiment, they derived an analytical formula for the efficiencies $e_{n}$ [3] (see Supplemental Material [15] and Ref. [3] for details),

$$
e_{n}=\frac{4 u u_{n}}{\left(u+u_{n}\right)^{2}} G(u) G\left(u_{n}\right)
$$

where the subscript $n$ denotes the diffraction order and

$$
G(x)=e^{2 x \zeta\left(\frac{1}{2}\right)} \prod_{l \geq 1} e^{\frac{-2 x}{\sqrt{l}}} \prod_{\substack{\left.l \geq 0 \\ l \neq x^{2}\right\}}}\left|\frac{1+\left(x / \sqrt{l+\left\{u^{2}\right\}}\right)}{1-\left(x / \sqrt{l+\left\{u^{2}\right\}}\right)}\right| .
$$

Here, $x=[x]+\{x\}$ with $\{x\}$ being the fractional part of $x$, the constant $\zeta\left(\frac{1}{2}\right)=-1.460354$ is given by the Riemann zeta function $\zeta(s)$, and $u_{n}=\sqrt{n+u^{2}}=\sin \left(\theta_{n} / 2\right) \sqrt{2 d / \lambda}$. Thus, $e_{n}$ not only depends on $\theta_{\text {in }}$ but also on the $n$ th-order diffraction angle $\theta_{n}$. The prediction by Eqs. (2) and (3) is in excellent agreement with a numerical solution that was later and independently reported by Kouznetsov and Oberst [7] to predict reflection and diffraction of ultracold-atoms scattering off an array of nanoscale ridges $[6,7]$.

Figure 1(b) shows the calculated efficiencies as a function of $u$ exhibiting three characteristic features: (i) in the limit $u \rightarrow 0$, the specular efficiency $e_{0}$ approaches unity, while the other efficiencies decay to zero; (ii) the smaller the diffraction order $n$, the higher the diffraction efficiency $e_{n}$; and (iii) all curves show pronounced minima at Rayleigh conditions (see [16] and references therein), which are illustrated by the vertical dashed lines in Fig. 1(b). When the incidence angle is equal to the $m$ th-order Rayleigh angle, $\theta_{\text {in }}=\theta_{R, m}$, and hence $u=u_{R, m}$, the $m$ th-order diffraction beam emerges parallel to the grating plane. Here, $u_{R, m}=\sin \left(\theta_{R, m} / 2\right) \sqrt{2 d / \lambda}=\sqrt{|m|}$.

A comprehensive experimental verification of the theoretical analysis has not been reported yet. In microwave propagation studies, the attenuation of a radio signal in urban areas caused by scattering from buildings is the main concern and has been measured [8,9]. Efficiencies of individual diffraction beams, however, have not been analyzed in that context. In the cold-atom diffraction experiments, on the other hand, specular reflection and first-order diffraction have been observed [17]. However, no quantitative analysis of nonspecular diffraction efficiencies has been reported, because the parameter range was limited to $u<1[6,7]$.

Furthermore, atom diffraction experiments exhibit an additional complication absent in scattering of electromagnetic waves. The dispersion interaction (van der WaalsCasimir-Polder interaction) between a neutral atom or molecule and the solid half planes can significantly obscure the phenomena of half-plane array diffraction. For an idealized array of infinitely thin half planes, an interaction-induced reduction of diffraction efficiencies was predicted $[7,18]$. In addition, any experimental implementation will exhibit some finite width of the half planes. Consequently, the interaction potential at the half-plane tops can lead to "quantum reflection" [19-25] of the atoms or molecules, resulting in increased efficiencies, which cover up the ideal half-plane edge diffraction. Quantum reflection from solids has been observed for ultracold atoms $[17,26]$ and Bose-Einstein condensates $[27,28]$ as 


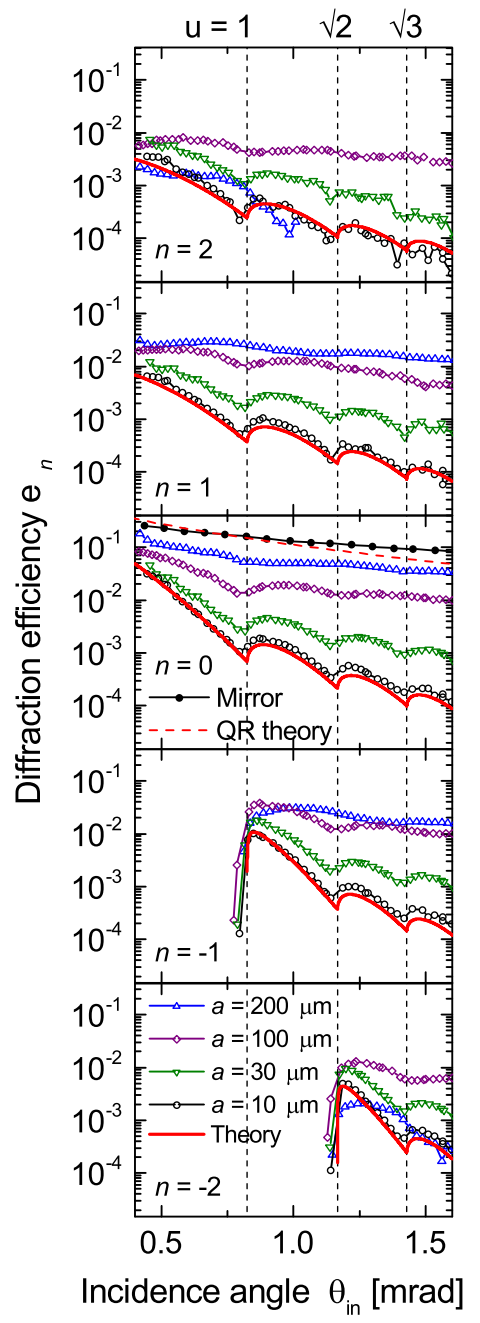

FIG. 3. Observed diffraction efficiencies (open symbols) for He atoms scattering from a square-wave grating compared with theory (red solid lines) calculated for an array of half planes. The efficiencies are plotted on a logarithmic scale as a function of incidence angle $\theta_{\text {in }}$ for diffraction orders $n=2,1,0,-1$, and -2 for different grating strip widths of 10, 30, 100, and $200 \mu \mathrm{m}$. The third graph also includes the reflection probability of He atoms from the blank photoresist (full black circles, labeled "mirror") as well as the calculated quantum reflection (QR) probability (red dashed line [39]). The dotted vertical lines indicate the Rayleigh incident conditions $\theta_{R, m}$ and $u_{R, m}$ for $m=-1,-2$, and -3 sequentially, where the $m$ th-order diffraction beam emerges from the grating plane. The corresponding $u_{R, m}$ values according to Eq. (1) are labeled atop the graph.

well as thermal-energy beams of atoms [29-32], molecules [33,34], and fragile helium clusters [35,36]. Furthermore, matter-wave diffraction by quantum reflection from a grating has also been observed with atoms, molecules, and clusters $[16,17,30,33-36]$, indicating the coherent nature of quantum reflection.

Here, we confirm the single-parameter model of wave scattering from a periodic half-plane array at grazingincidence conditions. We measure diffraction efficiencies of $\mathrm{He}$ atomic and $\mathrm{D}_{2}$ molecular beams reflecting off a square-wave grating of period $d=400 \mu \mathrm{m}$ composed of narrow strips of width $a=10,30,100$, or $200 \mu \mathrm{m}$. With decreasing $a$, the grating approaches the ideal periodic array of infinitely thin half planes. Given the $\lambda=136 \mathrm{pm}$ de Broglie wavelength of both $\mathrm{He}$ and $\mathrm{D}_{2}$ in our experiment, adjusting the incidence angle $\theta_{\text {in }}$ from 0.4 to $1.6 \mathrm{mrad}$ allows us to vary the $u$ parameter from 0.5 to almost 2 , thereby covering the conditions where three new additional diffraction orders emerge from the grating surface progressively.

Figure 2(a) shows a schematic of the experiment. The matter-wave diffraction apparatus has been described in detail before (see Supplemental Material [37] or previous publications [30,34]). In brief, it allows generation of intense continuous atomic or molecular beams exhibiting narrow velocity spread [38]. Tight beam collimation by three narrow slits reduces the angular peak width to $100 \mu \mathrm{rad}$ full width at half maximum. This, in combination with the precisely rotatable detector, makes it possible to observe high-resolution matter-wave diffraction patterns. For grazing-incidence conditions, the instrument allows us to resolve various diffraction orders despite the very large ratio of grating period to wavelength: $d / \lambda \simeq 3 \times 10^{6}$. An example angular spectrum (diffraction pattern) is shown in Fig. 2(c). In addition to the gratings, we prepared a sample of blank (not structured) photoresist of the same type and thickness $(1 \mu \mathrm{m})$, allowing us to compare the reflectivity measured with the gratings to the reflectivity of the unstructured photoresist.

The numbers above each peak in the angular pattern in Fig. 2(c) indicate the diffraction order $n$. By fitting each peak to a Gaussian, we determine the peak area $A_{n}$. Similarly, the incident beam area $A_{\text {in }}$ is determined from the angular profile measured when the grating is fully removed from the beam path. For each grating, we measured about 50 diffraction patterns like the one displayed in Fig. 2(d) over an incidence angle range $0.4<\theta_{\text {in }}<1.6 \mathrm{mrad}$. For each pattern, the $n$ th-order diffraction efficiencies $e_{n}=A_{n} / A_{\text {in }}$ were determined. Figure 3 shows the measured efficiencies as a function of the incident angle $\theta_{\text {in }}$ for five diffraction orders $n=2,1$, $0,-1$, and -2 .

For comparison, the Bogomolny-Schmit model prediction from Fig. 1(b) is plotted again in Fig. 3 (red solid lines). For the smallest strip width $a=10 \mu \mathrm{m}$, the agreement with the measured efficiencies appears to be very good for any $n$ including the specular beam $(n=0)$. Especially, the measured data exhibit the predicted hierarchy (the larger the $n$, the smaller the efficiency) and the pronounced minima at Rayleigh angles that represent the fingerprint of half-plane diffraction. Furthermore, for the smallest $u$ in our measurements $(u \simeq 0.5)$, not only $e_{0}$ but also $e_{1}$ and $e_{2}$ increase with decreasing $u$ in agreement with theory. Thus, we cannot directly check the prediction of $e_{0}$ approaching unity and $e_{n}(n>0)$ vanishing in the 


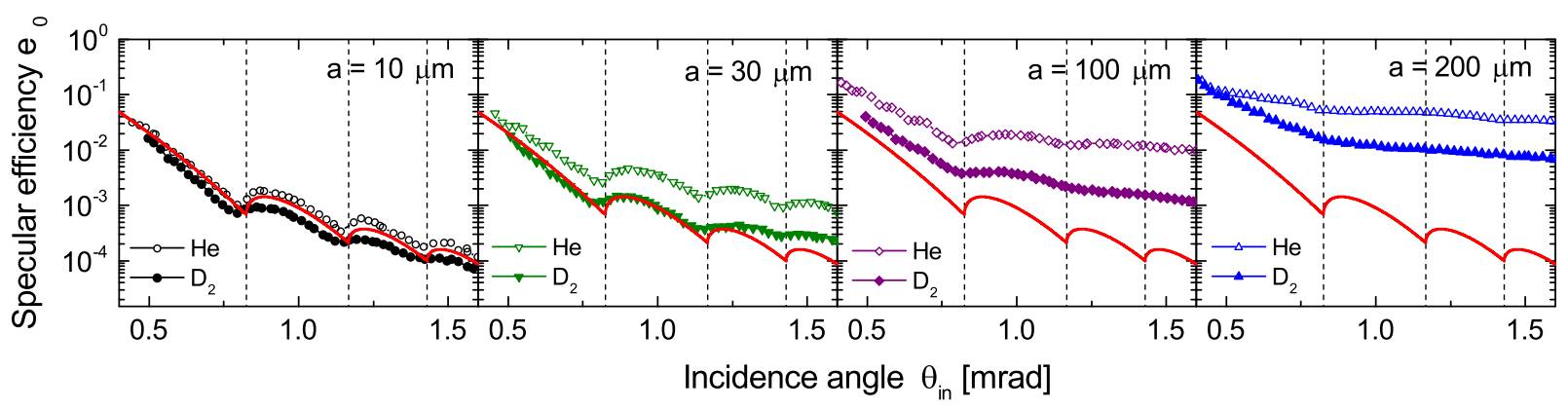

FIG. 4. Specular beam efficiencies observed for He and $\mathrm{D}_{2}$ scattering from the gratings of different strip widths $a$ as indicated. The efficiencies are plotted on a logarithmic scale as a function of incidence angle $\theta_{\text {in. }}$. As in Fig. 3, red lines represent the prediction by Eqs. (2) and (3), and the dotted vertical lines indicate Rayleigh conditions $\theta_{R, m}$ and $u_{R, m}$ of $m$ th-order beam emergence for $m=-1,-2$, and -3 .

limit $u \rightarrow 0$. However, by applying the reciprocity principle, we conclude from the behavior of $e_{-1}$ and $e_{-2}$ that $e_{1}$ and $e_{2}$ vanish when $u$ gets close to zero [40]. The only discrepancy between experiment and theory found for $a=$ $10 \mu \mathrm{m}$ is the measured efficiencies being slightly larger than the calculated ones, mainly for parts of the data for $n=0$ and $n=-1$.

As $a$ increases, the diffraction efficiencies are found to increase dramatically (except for $e_{ \pm 2}$ at $a=200 \mu \mathrm{m}$ ) and decay much slower with increasing $u$. Concurrently, the pronounced minima at Rayleigh conditions smear out and are indiscernible for $a=200 \mu \mathrm{m}$. In the third graph of Fig. 3, the specular reflectivity data measured with the blank photoresist have been added for comparison. Its slope resembles the specular efficiency data observed for $a=200 \mu \mathrm{m}$, but it is larger by an approximately constant factor of 2-3. This behavior is consistent with the assumption that the total reflection probability scales with the reflecting area atop the strips. The reflecting area of the blank surface is twice the one of the $a=200 \mu \mathrm{m}$ grating for which the totally reflected flux is branched among the specular and diffraction peaks. In previous work, we have identified quantum reflection as the process giving rise to coherent reflection from a grating [30] or a blank surface [31] for the present grazing-incidence conditions. The red dashed line presents the calculated quantum reflection probability (see Supplemental Material [39] for details).

For $a>10 \mu \mathrm{m}$, quantum reflection from the flat tops of the grating strips accounts for the increased diffraction efficiencies. According to the classical FraunhoferKirchhoff diffraction theory for an amplitude grating, $e_{0}$ and $e_{ \pm 1}$ increase continuously when the ratio $a / d$ increases towards $1 / 2$ [1]. The second-order diffraction efficiencies, on the contrary, exhibit a maximum for $a / d=0.25$ and vanish at $a / d=0.5$. These features are well manifested in our results; with $a$ increasing to $d / 2$ we find $e_{0}$ approaching $40 \%$ of the blank surface reflection probability, and we observe very small values of $e_{ \pm 2}$ for $a=200 \mu \mathrm{m}$. This behavior indicates that the helium atoms are reflected from the grating dominantly by half-plane diffraction and quantum reflection for $a=10$ and $200 \mu \mathrm{m}$, respectively, with a transition between the two regimes for intermediate strip widths.

To check this interpretation, we repeated the experiment with a $\mathrm{D}_{2}$ molecular beam of identical de Broglie wavelength. $\mathrm{D}_{2}$ has a 3.9 times larger polarizability than He. Therefore, we expect the stronger van der Waals interaction of $\mathrm{D}_{2}$ with the grating strips to result in (i) reduced quantum reflection $[30,33]$ and (ii) attenuation of the half-plane array diffraction efficiencies $[7,18]$. Figure 4 shows a comparison of the observed $\mathrm{D}_{2}$ and $\mathrm{He}$ specular reflection efficiencies $e_{0}$. Both effects are clearly visible in the data. For the smallest strip width $a=10 \mu \mathrm{m}$, the $\mathrm{D}_{2}$ data are about $50 \%$ less than the He data and appear now below the theory curve. At $a=200 \mu \mathrm{m}$, the $\mathrm{D}_{2}$ specular efficiency is found to be only about $20 \%$ of the corresponding He data for large incidence angle, while both datasets converge in the small angle limit as expected.

In our present experiment, quantum reflection probabilities are larger than those due to half-plane array diffraction. This is due to the fact that we have used ground-state $\mathrm{He}$ atoms and $\mathrm{D}_{2}$ molecules, which interact weakly with a surface, in combination with a rather sparse grating with a relatively large period of $d=0.4 \mathrm{~mm}$. Reduction of the latter is easy to implement and will make it possible to increase the reflectivity significantly. Furthermore, halfplane array reflection results from diffraction at the halfplane edges. Hence, unlike classical reflection and quantum reflection, it occurs independent of the interaction potential between the atom or molecule and the half-plane surface. This is analogous to diffraction of atoms and molecules by a nanoscale transmission grating [41], where localization of the wave function by nanoscale slits and the corresponding decrease (increase) of uncertainly in real space (momentum space) result in diffracted beams. While van der Waals interaction of the atoms and molecules with the grating bars (i.e., the half planes in the present experiment) influences the diffraction intensities [7,42], it is not the origin of diffraction [43]. Consequently, half-plane diffraction can be applied in 
future experiments to weakly bound molecules, such as the $\mathrm{He}$ dimer and trimer [48], for which classical and quantum reflection are inhibited or inefficient [35].

In conclusion, we measured diffraction efficiencies up to the second order of He atoms with $136 \mathrm{pm}$ de Broglie wavelength scattering from a $400-\mu \mathrm{m}$-period square-wave grating at grazing incidence. By varying the grating strip width from $200 \mu \mathrm{m}$ down to $10 \mu \mathrm{m}$, we observe a transition from quantum reflection to the regime where edge diffraction from half planes dominates. Our data provide a test bench for models of quantum reflection from microstructured surfaces that need to account for half-plane edge diffraction. In addition, our results provide the first experimental validation, including $n \neq 0$ diffraction efficiencies, of the single-parameter model of diffraction from a halfplane array in the semiclassical limit, which is used in diverse fields such as radio-wave propagation in urban areas (Walfisch-Bertoni model) or pseudointegrable polynomial billiards (Bogomolny-Schmit model).

This work was supported by the Basic Science Research Program through the National Research Foundation of Korea (NRF) funded by the Ministry of Science, ICT, and Future Planning (Nos. NRF-2015R1A2A2A01005458, NRF2017R1A2B4008226, and NRF-2017H1A2A1042369).

J. H. L. and L. Y. K. contributed equally to this work.

*wschoell@fhi-berlin.mpg.de

'zhao@unist.ac.kr

[1] M. Born and E. Wolf, Principles of Optics, 6th ed. (Cambridge University Press, Cambridge, England, 1997).

[2] P. J. Richens and M. V. Berry, Physica (Amsterdam) 2D, 495 (1981).

[3] E. Bogomolny and C. Schmit, Nonlinearity 16, 2035 (2003).

[4] E. Bogomolny and C. Schmit, Phys. Rev. Lett. 92, 244102 (2004).

[5] E. Bogomolny, B. Dietz, T. Friedrich, M. Miski-Oglu, A. Richter, F. Schäfer, and C. Schmit, Phys. Rev. Lett. 97, 254102 (2006).

[6] H. Oberst, D. Kouznetsov, K. Shimizu, J. I. Fujita, and F. Shimizu, Phys. Rev. Lett. 94, 013203 (2005).

[7] D. Kouznetsov and H. Oberst, Phys. Rev. A 72, 013617 (2005).

[8] L. E. Vogler, Radio Sci. 17, 1541 (1982).

[9] J. Walfisch and H.L. Bertoni, IEEE Trans. Antennas Propag. 36, 1788 (1988).

[10] H. H. Xia and H. L. Bertoni, IEEE Trans. Antennas Propag. 40, 170 (1992).

[11] J. F. Carlson and A. E. Heins, Q. Appl. Math. 4, 313 (1947).

[12] A. E. Heins and J. F. Carlson, Q. Appl. Math. 5, 82 (1947).

[13] B. A. Lengyel, J. Appl. Phys. 22, 265 (1951).

[14] J. J. Brady, M. D. Pearson, and S. R. Peoples, J. Appl. Phys. 23, 964 (1952).
[15] See the section "Numerical calculation of the asymptotic formula" in the Supplemental Material at http://link.aps.org/ supplemental/10.1103/PhysRevLett.122.040401.

[16] B. S. Zhao, G. Meijer, and W. Schöllkopf, Phys. Rev. Lett. 104, 240404 (2010).

[17] F. Shimizu and J. Fujita, J. Phys. Soc. Jpn. 71, 5 (2002).

[18] D. Kouznetsov, H. Oberst, A. Neumann, Y. Kuznetsova, K. Shimizu, J.-F. Bisson, K. Ueda, and S. R. J. Brueck, J. Phys. B 39, 1605 (2006).

[19] R. B. Doak and A. V. G. Chizmeshya, Europhys. Lett. 51, 381 (2000).

[20] A. Mody, M. Haggerty, J. M. Doyle, and E. J. Heller, Phys. Rev. B 64, 085418 (2001).

[21] H. Friedrich, G. Jacoby, and C. G. Meister, Phys. Rev. A 65, 032902 (2002).

[22] B. A. Stickler, U. Even, and K. Hornberger, Phys. Rev. A 91, 013614 (2015).

[23] S. Miret-Artés and E. Pollak, J. Phys. Chem. Lett. 8, 1009 (2017).

[24] E. Galiffi, C. Sünderhauf, M. DeKieviet, and S. Wimberger, J. Phys. B 50, 095001 (2017).

[25] J. Petersen, E. Pollak, and S. Miret-Artés, Phys. Rev. A 97, 042102 (2018).

[26] F. Shimizu, Phys. Rev. Lett. 86, 987 (2001).

[27] T. A. Pasquini, Y. Shin, C. Sanner, M. Saba, A. Schirotzek, D. E. Pritchard, and W. Ketterle, Phys. Rev. Lett. 93, 223201 (2004).

[28] T. A. Pasquini, M. Saba, G.-B. Jo, Y. Shin, W. Ketterle, D. E. Pritchard, T. A. Savas, and N. Mulders, Phys. Rev. Lett. 97, 093201 (2006).

[29] V. Druzhinina and M. DeKieviet, Phys. Rev. Lett. 91, 193202 (2003).

[30] B. S. Zhao, S. A. Schulz, S. A. Meek, G. Meijer, and W. Schöllkopf, Phys. Rev. A 78, 010902 (2008).

[31] B. S. Zhao, H. C. Schewe, G. Meijer, and W. Schöllkopf, Phys. Rev. Lett. 105, 133203 (2010).

[32] A. R. Barnea, B. A. Stickler, O. Cheshnovsky, K. Hornberger, and U. Even, Phys. Rev. A 95, 043639 (2017).

[33] B. S. Zhao, W. Zhang, and W. Schöllkopf, Sci. Adv. 2, e1500901 (2016).

[34] W. Zhang, J. H. Lee, H. A. Kim, B. G. Jin, B. J. Kim, L. Y. Kim, B. S. Zhao, and W. Schöllkopf, Chem. Phys. Chem 17, 3670 (2016).

[35] B. S. Zhao, G. Meijer, and W. Schöllkopf, Science 331, 892 (2011).

[36] B. S. Zhao, W. Zhang, and W. Schöllkopf, Mol. Phys. 111, 1772 (2013).

[37] See the section "Atomic and molecular beam apparatus" in the Supplemental Material at http://link.aps.org/ supplemental/10.1103/PhysRevLett.122.040401.

[38] L. W. Bruch, W. Schöllkopf, and J. P. Toennies, J. Chem. Phys. 117, 1544 (2002).

[39] See the section "Calculated quantum reflection probability" in the Supplemental Material at http://link.aps.org/ supplemental/10.1103/PhysRevLett.122.040401.

[40] According to the reciprocity theorem, $e_{n}$ for $n>0$ near $u=0$ are necessarily the same as $e_{-n}$ near the Rayleigh angles $u=u_{R,-n}$. The observed $e_{-1}$ and $e_{-2}$ values approach zero at those Rayleigh conditions (see the lower two graphs in Fig. 3). 
[41] W. Schöllkopf and J. P. Toennies, Science 266, 1345 (1994).

[42] R. E. Grisenti, W. Schöllkopf, J.P. Toennies, G. C. Hegerfeldt, and T. Köhler, Phys. Rev. Lett. 83, 1755 (1999).

[43] See the section "Qualitative picture of half-plane array diffraction" in the Supplemental Material at http://link.aps .org/supplemental/10.1103/PhysRevLett.122.040401, which includes Refs. [44-47].

[44] R. E. Grisenti, W. Schöllkopf, J. P. Toennies, G. C. Hegerfeldt, T. Köhler, and M. Stoll, Phys. Rev. Lett. 85, 2284 (2000).

[45] G. C. Hegerfeldt and T. Köhler, Phys. Rev. A 57, 2021 (1998).
[46] G. C. Hegerfeldt and T. Köhler, Phys. Rev. A 61, 023606 (2000).

[47] J. R. Manson, in Helium Atom Scattering from Surfaces, edited by E. Hulpke, Springer Series in Surface Science, Vol. 27 (Springer, Berlin, 1992), pp. 173-205.

[48] See the section "Half-plane array diffraction of helium trimers" in the Supplemental Material http://link.aps.org/ supplemental/10.1103/PhysRevLett.122.040401, which includes Ref. [49].

[49] F. Shimizu and J. I. Fujita, Phys. Rev. Lett. 88, 123201 (2002). 